\title{
What intrinsic factors influence responsiveness to acupuncture in pain?: a review of pre-clinical studies that used responder analysis
}

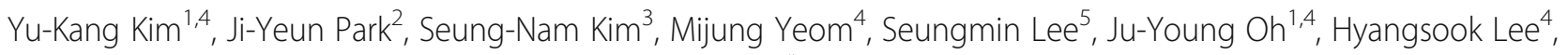
Younbyoung Chae ${ }^{4}$, Dae-Hyun Hahm ${ }^{4}$ and Hi-Joon Park ${ }^{4^{*}}$

\begin{abstract}
Background: Not many studies have investigated individual sensitivity to acupuncture. To explore the intrinsic factors related to individual responses to acupuncture, we reviewed published pre-clinical studies using responder analysis on pain.

Methods: We searched the PubMed and EMBASE databases to June 2015. We included pre-clinical reports describing responders and non-responders to anti-nociceptive and analgesic effects of acupuncture in animal study. We identified the potential intrinsic factors which might be related with the response to acupuncture.

Results: Totally, 216 potentially relevant articles were retrieved and 14 studies met our inclusion criteria. Rat $(n=1348)$ and rabbit $(n=56)$ were used, and only electroacupuncture (EA) was applied as an intervention. Results showed that high levels of cholecystokinin-8 and receptors were associated with poor responsiveness to EA. Endogenous opioids including $\beta$-endorphin and met-enkephalin, descending inhibitory norepinephrine and serotonin system, and hypothalamic 5'-AMP-activated protein kinase seemed to be associated with high-level responses. Spinal levels of neurotransmitters and pro-inflammatory cytokines were also differentially expressed depending on the EA sensitiveness. In the central nervous system, hypothalamus, periaqueductal grey, pituitary gland, and spinal cord were suggested to be involved in the EA responsiveness. Identified individual variations did not seem to be accidental, as the responsiveness to EA was replicated over time. However, methodological issues such as reproducibility, cut-off criteria, and clinical relevance need to be further elaborated.
\end{abstract}

Conclusion: Our study suggests that the identification of the biological factors differentiating responders from non-responders is necessary and it may aid in understanding how acupuncture modulates pain.

Keywords: Acupuncture, Responder, Non-responder, Individual difference, Cholecystokinin

\section{Background}

Pain is the most prevalent reason patients seek medical attention in many developed countries $[1,2]$. It is a chief complaint in numerous disorders that hinders the individual's daily activities and functions [3, 4]. Analgesics can help alleviate such pain but individual sensitivity to pain and individual responsiveness to pharmaceuticals

\footnotetext{
* Correspondence: acufind@khu.ac.kr

${ }^{4}$ Acupuncture \& Meridian Science Research Center, Kyung Hee University, 26 Kyungheedae-ro, Dongdaemoon-gu, Seoul 02447, Republic of Korea Full list of author information is available at the end of the article
}

vary considerably even among patients with the same conditions. In the clinic, for example, although morphine has great analgesic properties, some patients experience inadequate analgesia and demand increased dosage or concurrently suffer from significant side effects [5]. These inter-individual differences in the factors causing pain and in the pain mechanisms per se, are clearly in play and a more individualistic approach, which focuses on the responder and non-responder differences, may be useful in the development of novel analgesic treatment [6-8]. 
Acupuncture, the stimulation of specific acupoints on the body with acupuncture needles, is widely used to relieve persistent pain and to treat many disorders [9-12]. An important feature of acupuncture is that the chosen acupoints and the preferred type of manipulation reflect patients' individual characteristics and/or those of their diseases. Intrinsic patient factors are, therefore, at the forefront when doctors plan overall treatment [13].

For decades, researchers have tried to identify what intrinsic factors may constitute the specific effects of acupuncture. A recent innovative brain imaging study showed that acupuncture-induced analgesia of migraine involves the intrinsic functional connectivity of the right frontoparietal network [14]. However other than this study, most of the previous research has only focused on elucidating acupuncture's therapeutic effects, while individual responsiveness was less considered [15]. Analysis of both responders and non-responders would reap the benefits additional to investigating acupuncture outcomes and developing therapeutic effects.

A few preclinical or human studies have provided significant insight into the differences between responders or non-responders. Electroacupuncture (EA) afforded either excellent or almost no effects in human or animals, i.e., it produced beneficial effects in approximately $70 \%$ of subjects and resulted in little changes in the other 30\%. [16-18]. Low responsiveness to EA effects was known to be associated with changes in cholecystokinin (CCK) levels [19]. It was also suggested that the degree of responsiveness to acupuncture effects was associated with different neural activation by EA in periaqueductal grey (PAG) or hypothalamus [20, 21]. Thus, further work on the mechanisms involved and specific regulation of intrinsic factors related with high or low responsiveness might allow the therapeutic effects of acupuncture to be better predicted. However, to date, no systematic study has distinguished between responders and non-responders when acupuncture was used to modulate pain.

In this review, we reviewed the relevant published literature of pre-clinical studies to define high and low responsiveness of EA to pain and identify trends in the associated factors, regions, or mechanisms of responders and non-responders.

\section{Methods}

\section{Search strategy}

We searched the PubMed and EMBASE databases to June 2015. The search term was "acupuncture and (responder* or non-responder*)." Additionally, publications in the reference lists of retrieved papers and relevant reviews were manually retrieved.

\section{Study selection}

We first screened the records by title and abstract; we included original English-language reports on animal experiments discriminating responders from nonresponders. The exclusion criteria were: 1) use of a language other than English, 2) irrelevance in terms of acupuncture treatment, 3) lack of baseline or outcome data, 4) definition of responders using only a survey or a questionnaire, 5) the absence of any distinction between responders and non-responders to acupuncture, 6) the absence of a focus on differences between responders and non-responders, and 7) not conducted in animal models. We included any form of acupuncture and any animal model. As we sought to define the features of acupuncture associated with a response or a non-response, all included studies had to distinguish between responders and non-responders. Any ambiguities were discussed by three reviewers (Kim YK, Park JY, and Park HJ) until unanimous consensus was attained.

\section{Data extraction}

We recorded the first author's name, the year of publication, the country in which the work was performed, the animal used (sex and species), the sample size, whether the animals were healthy or diseased, the type of acupuncture applied, the EA parameters (if relevant), the acupoints used, the cut-offs for identification as responders/non-responders, the numbers of responders and non-responders, the outcomes of any behavioral tests applied, target molecules, regions of interest, the defined functions of target molecules, and the outcomes of EA-sensitivity analysis. Data extraction was performed by one author (Kim YK), but two other authors (Park JY, Park HJ) double-checked this work.

\section{Quality assessment}

The included publications were independently assessed by two authors (Kim YK, Park HJ) in terms of the methodological quality using the 9-item checklist modified from the CAMARADES checklist [22, 23]: (1) peerreviewed publication; (2) detailed statement of acupuncture procedure; (3) objective behavior test applied to classify responder or non-responder; (4) statement of cut-off values for dividing responder or non-responder; (5) notification of the ratio of responder or nonresponder to the total; (6) blinded assessment of outcome; (7) reproducibility of EA sensitiveness; (8) compliance with animal welfare regulations; and (9) statement of potential conflict of interests. Each study was recorded a sum quality score out of a possible total of 9 points. Discrepancies were resolved after discussion between the two authors (Kim YK, Park HJ). 


\section{Results}

\section{An overview of the study}

Figure 1 shows how reports were selected. We initially identified 106 and 192 relevant publications in PubMed and EMBASE respectively, and additionally added 14 works via manual searching. Among these 312 records, 96 duplicated articles were removed. We excluded 194 of these 216 papers after screening the abstracts and titles. Ultimately, 22 publications were fully evaluated. We subsequently excluded eight of these because responders were not classified (three studies) or studies did not explore factors associated with individual variations (five studies). Finally, 14 studies met our inclusion criteria.

\section{Characteristics of included studies}

Table 1 lists the characteristics of the included studies. A total of 1348 rats and 52 rabbits were used. All studies featured EA of low frequency $(1-3 \mathrm{~Hz}$, nine studies), medium frequency $(15 \mathrm{~Hz}$, one study), or high frequency (100 Hz, five studies). All studies electrically connected acupoint ST36 with acupoint SP6 (six studies), or the adjacent bilateral or ipsilateral ST36 acupoints (six studies). One study used acupoint TE18 in the rabbit. Only three studies included pain models including neuropathic pain [18], incisional pain [24], and inflammatory pain [25] in their evaluations of pain levels; 13 studies employed normal animals.

\section{Study quality}

The quality score of the included studies ranged from 2 to 7 out of a total 9 points. Of the 14 studies, 3 studies got 2 points [19-21], 3 studies got 3 points [17, 26, 27],
3 studies got 4 points [28-30], 1 study got 5 points [31], 2 studies got 6 points $[24,25]$, one study got 7 points [18], and one study got 8 points [32] (Table 2). All 14 studies were published in peer reviewed journals. Six studies described detailed acupuncture procedure [24-26, 28, 30, 32]. Six studies reported objective behavior test applied to classify responder of nonresponder [18, 25, 27, 29, 31, 32]. All 14 studies notified cut-off value for dividing responder or non-responder. None of the studies mentioned blinded assessment of outcome. Two studies confirmed reproducibility of EA sensitiveness [18, 32]. Six studies reported compliance with animal welfare regulations [18, 24, 25, 28, 31, 32]. Four studies contained statement of potential conflict of interests [18, 24, 31, 32].

\section{Cut-off values for EA responses}

Thirteen studies used the tail flick latency (TFL) to distinguish between EA responders and non-responders; one employed the paw pressure threshold [25]. However, the criteria used to separate responders and nonresponders in terms of EA sensitivity were very different. In 10 studies, percentage changes in pain behavior (from baseline) were calculated: cut-off values in the responder ranged $10-150 \%$ increase in TFL or the pressure pain threshold, and those in the non-responders were $0-50 \%$. Three studies defined responders as animals exhibiting statistically significant increases in TFL $(p<0.05)$ compared with baseline $[20,21,28]$. One study defined a non-responder as an animal in which the TFL change was less than the mean value plus three standard deviations [24]. All criteria presented were shown in Table 3, and the proportion of responders according to the

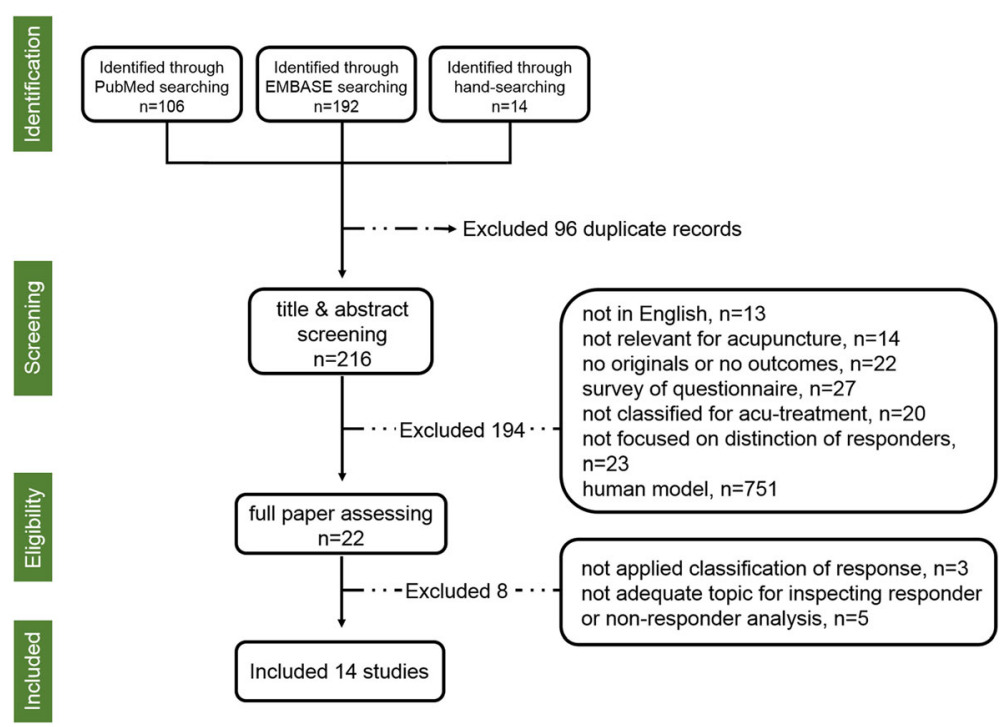

Fig. 1 Flow diagram of the review 
Table 1 Experimental index for electroacupuncture

\begin{tabular}{|c|c|c|c|c|c|}
\hline Author (Year, Country) & Animal (sex) & Sample size & Condition & Acupoints (side) & $\begin{array}{l}\text { EA Parameters: frequency, } \\
\text { amplitude, duration }\end{array}$ \\
\hline Kim $(2014$, Korea $)$ & rats $(M)$ & 18 & Nor & ST36-subST36 (n.r.) & $2 \mathrm{~Hz}, 0.2-0.3 \mathrm{~mA}, 20 \mathrm{~min}$ \\
\hline Wang (2012, China) & rats $(M)$ & 170 & Nor & ST36-SP6 (B) & $\begin{array}{l}2 \mathrm{~Hz} \text { or } 100 \mathrm{~Hz} \text { respectively, } \\
0.5 / 1.0 / 1.5 \mathrm{~mA}, 30 \mathrm{~min}\end{array}$ \\
\hline Fais (2012, Brazil) & rats $(M)$ & 48 & Incisional pain & ST36-SP6 (B) & $2 \mathrm{~Hz}, 1.4-1.5 \mathrm{~mA}, 20 \mathrm{~min}$ \\
\hline Gao (2007, China) & rats $(M)$ & 18 & Nor & ST36-subST36 (B) & $1 \mathrm{~Hz}, 3.5-5 \mathrm{~V}, 60 \mathrm{~min}$ \\
\hline Kim (2007, Korea) & $\begin{array}{l}\text { rats }(M) \\
\text { CCK-AR KO rats (M) }\end{array}$ & 38 & Nor / Neur. pain & ST36-subST36 (n.r.) & $2 \mathrm{~Hz}, 0.2-0.3 \mathrm{~mA}, 20 \mathrm{~min}$ \\
\hline Ko $(2006$, Korea $)$ & rats $(M)$ & 12 & Nor & ST36-subST36 (n.r.) & $100 \mathrm{~Hz}, 0.2-0.3 \mathrm{~mA}, 20 \mathrm{~min}$ \\
\hline Sekido (2003, Japan) & rats $(M)$ & 95 & Nor / Paw inflam & ST36-subST36 (L) & $3 \mathrm{~Hz}, 1 / 2 / 3 \mathrm{~mA}, 60 \mathrm{~min}$ \\
\hline Lee (2002, Korea) & rats $(M)$ & 12 & Nor & ST36-subST36 (n.r.) & $2 \mathrm{~Hz}, 0.2-0.3 \mathrm{~mA}, 15 \mathrm{~min}$ \\
\hline Liu (1999, China) & rats $(F)$ & 19 & Nor & ST36-SP6 (B) & $100 \mathrm{~Hz}, 1 / 2 / 3 \mathrm{~mA}, 30 \mathrm{~min}$ \\
\hline Tian (1998, China) & rats $(F)$ & 193 & Nor & ST36-SP6 (n.r.) & $100 \mathrm{~Hz}, 1 / 2 / 3 \mathrm{~mA}, 30 \mathrm{~min}$ \\
\hline Tang (1997, China) & rats $(F)$ & 215 & Nor & ST36-SP6 (B) & $100 \mathrm{~Hz}, 1 / 2 / 3 \mathrm{~mA}, 30 \mathrm{~min}$ \\
\hline Takeshige (1993, Japan) & $\begin{array}{l}\text { rats }(M) \text { and } \\
\text { rabbits (n.r.) }\end{array}$ & $\begin{array}{l}402 \text { rats and } \\
52 \text { rabbits }\end{array}$ & Nor & $\begin{array}{l}\text { Rats: ST36 (n.r.) } \\
\text { Rabbits: TE18 (n.r.) }\end{array}$ & $\begin{array}{l}1 \mathrm{~Hz} \text {, intensity to cause muscle } \\
\text { contraction, } 30 \mathrm{~min} \text {, } \\
45 \mathrm{~min} \text { or } 60 \mathrm{~min}\end{array}$ \\
\hline Takeshige (1992, Japan) & rats $(M)$ & 80 & Nor & ST36 (n.r.) & $\begin{array}{l}1 \mathrm{~Hz} \text {, intensity to cause muscle } \\
\text { contraction, n.r. }\end{array}$ \\
\hline $\operatorname{Han}(1985$, China) & rats (n.r.) & 28 & Nor & ST36-SP6 (n.r.) & $15 \mathrm{~Hz}, 3 \mathrm{~V}, 10 \mathrm{~min}$ \\
\hline
\end{tabular}

$B$ bilateral, CCK-AR KO cholecystokinin A receptor knockout, $F$ female; Incisional pain, mechanical hyperalgesia induced by $1 \mathrm{~cm}$ longitudinal incision through skin and fascia and stitches on right hind paw; $M$ male, $L$ left; Neur. pain neuropathic pain; Nor normal, n.r. not reported; Paw inflam, carrageenan-induced inflammation on the paw; subST36, $5 \mathrm{~mm}$ distal from ST36

Table 2 Risk of bias of the included studies

\begin{tabular}{lllllllllll}
\hline Author (Year) & A & B & C & D & E & F & G & H & I & Total \\
\hline Kim (2014) & $\sqrt{ }$ & & $\sqrt{ }$ & & $\sqrt{ }$ & & & $\sqrt{ }$ & $\sqrt{ }$ & 5 \\
Wang (2012) & $\sqrt{ }$ & $\sqrt{ }$ & $\sqrt{ }$ & & $\sqrt{ }$ & $\sqrt{ }$ & $\sqrt{ }$ & $\sqrt{ }$ & $\sqrt{ }$ & 8 \\
Fais (2012) & $\sqrt{ }$ & $\sqrt{ }$ & & & $\sqrt{ }$ & $\sqrt{ }$ & & $\sqrt{ }$ & $\sqrt{ }$ & 6 \\
Gao (2007) & $\sqrt{ }$ & $\sqrt{ }$ & & & $\sqrt{ }$ & & & $\sqrt{ }$ & & 4 \\
Kim (2007) & $\sqrt{ }$ & & $\sqrt{ }$ & & $\sqrt{ }$ & $\sqrt{ }$ & $\sqrt{ }$ & $\sqrt{ }$ & $\sqrt{ }$ & 7 \\
Ko (2006) & $\sqrt{ }$ & & $\sqrt{ }$ & & $\sqrt{ }$ & $\sqrt{ }$ & & & & 4 \\
Sekido (2003) & $\sqrt{ }$ & $\sqrt{ }$ & $\sqrt{ }$ & & $\sqrt{ }$ & $\sqrt{ }$ & & $\sqrt{ }$ & & 6 \\
Lee (2002) & $\sqrt{ }$ & & & & $\sqrt{ }$ & $\sqrt{ }$ & & & & 3 \\
Liu (1999) & $\sqrt{ }$ & $\sqrt{ }$ & & & $\sqrt{ }$ & & & & & 3 \\
Tian (1998) & $\sqrt{ }$ & & $\sqrt{ }$ & & $\sqrt{ }$ & & & & & 3 \\
Tang (1997) & $\sqrt{ }$ & $\sqrt{ }$ & & & $\sqrt{ }$ & $\sqrt{ }$ & & & & 4 \\
Takeshige (1993) & $\sqrt{ }$ & & & & $\sqrt{ }$ & & & & & 2 \\
Takeshige (1992) & $\sqrt{ }$ & & & & $\sqrt{ }$ & & & & \\
Han (1985) & $\sqrt{ }$ & & & & $\sqrt{ }$ & & & & & 2 \\
\hline
\end{tabular}

Studies fulfilling the criteria of: A: peer reviewed publication; B: detailed statement of acupuncture procedure; C: objective behavior test applied to classify responder or non-responder; D: blinded assessment of outcome $\mathrm{E}$ : statement of cut-off value for dividing responder or non-responder; F: notification of the ratio of responder or non-responder to the total; G: reproducibility of EA sensitiveness; $\mathrm{H}$ : compliance with animal welfare regulations; I: statement of potential conflict of interests various criteria are shown in Additional file 1: Fig. S1, in the case of provided information available.

\section{Reproducibility of responsiveness to the anti-nociceptive or analgesic effects of EA}

Wang et al. found that EA variance was maintained when TFL was evaluated on two successive days in a physiological state [32]. Kim et al. confirmed that responders exhibiting normal EA-mediated anti-nociception were consistently more sensitive to EA-induced analgesia in a model of neuropathic pain than were non-responders [18]. However, Sekido et al. reported different findings: $50 \%$ of normal rats were EA-responders, but all animals (thus both responders and non-responders) were susceptible to EA-induced analgesia after induction of carrageenan-mediated inflammatory pain [25].

\section{Intrinsic factors associated with a poor response to acupuncture}

Six studies suggested that the level of CCK or the receptor thereof were related with no or a low response. Han et al. showed that injection of an antibody against the CCK-octapeptide (CCK-8) changed an EA non-response into an apparent response [19], and Tang et al. confirmed that inhibition of brain CCK synthesis rendered non-responders responders [30]. Liu et al. showed that the spinal perfusates of non-responders exhibited a higher level of CCK immunoreactivity than did that of 
Table 3 EA response criteria and associated data

\begin{tabular}{|c|c|c|c|c|}
\hline Author (Year) & $\begin{array}{l}\text { Behavior test } \\
\text { for cut off }\end{array}$ & $\begin{array}{l}\text { EA } \\
\text { frequency }\end{array}$ & $\begin{array}{l}\text { EA response criteria: Changes from the bas } \\
(n, \%=\text { proportion of responder or non-res }\end{array}$ & \\
\hline \multirow[t]{2}{*}{ Kim et al. (2014) } & TFL & LF & & \\
\hline & & & NR (8, n.a.) & R (10, n.a.) \\
\hline
\end{tabular}

Wang et al. (2012) TFL LF

HF

a) \begin{tabular}{l|l}
$\stackrel{\mathrm{NR}(7,8.8 \%)}{R}(54,67.5 \%)$ & $\mathrm{HR}(\mathbf{1 9}, \mathbf{2 3 . 8 \% )}$ \\
\hline $0 \%$
\end{tabular}

Kim et al. (2007) TFL LF

\begin{tabular}{l|ll}
\hline NR $(7,38.9 \%)$ & $\mathrm{R}(11,61.1 \%)$ \\
\hline $30 \%$
\end{tabular}

Ko et al. (2006) TFL HF

\begin{tabular}{l|ll}
\hline a) & NR $(6,50.0 \%)$ & $\mathrm{R}(6,50.0 \%)$ \\
\hline
\end{tabular}

Lee et al. (2002) TFL LF

\begin{tabular}{l|ll}
$\longleftrightarrow$ NR $(5,41.7 \%)$ & $\mathrm{R}(7,58.3 \%)$ \\
\hline
\end{tabular}

Liu et al. (1999) TFL HF

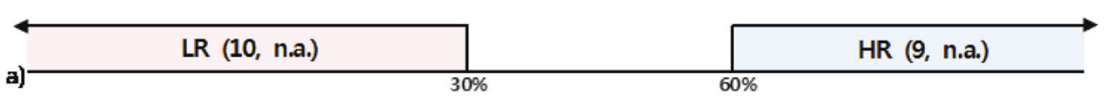

Tian et al. (1998) TFL HF

\begin{tabular}{|l|l|}
\hline LR (47, n.a.) & HR (43, n.a.) \\
\hline
\end{tabular}

Tang et al. (1997) TFL HF

\begin{tabular}{l|lll} 
LR & (discarded) & HR (14, 38.9\%) \\
\hline $30 \%$ &
\end{tabular}

Han et al. (1985) TFL IF

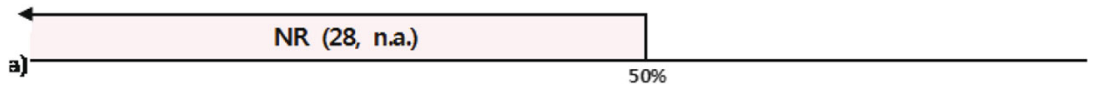

Sekido et al. (2003) PPT LF

\begin{tabular}{l|ll}
\hline $\mathrm{NR}(14,48.3 \%)$ & $\mathrm{R}(15,51.7 \%)$ \\
\hline \multicolumn{2}{|c|}{} \\
\hline \multicolumn{2}{|c|}{ a) }
\end{tabular}

Gao et al. (2007) TFL LF

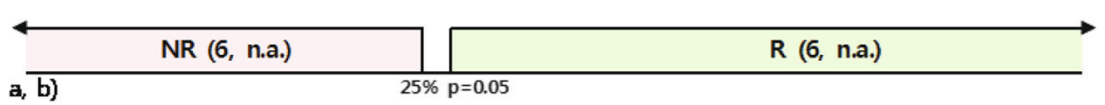


Table 3 EA response criteria and associated data (Continued)

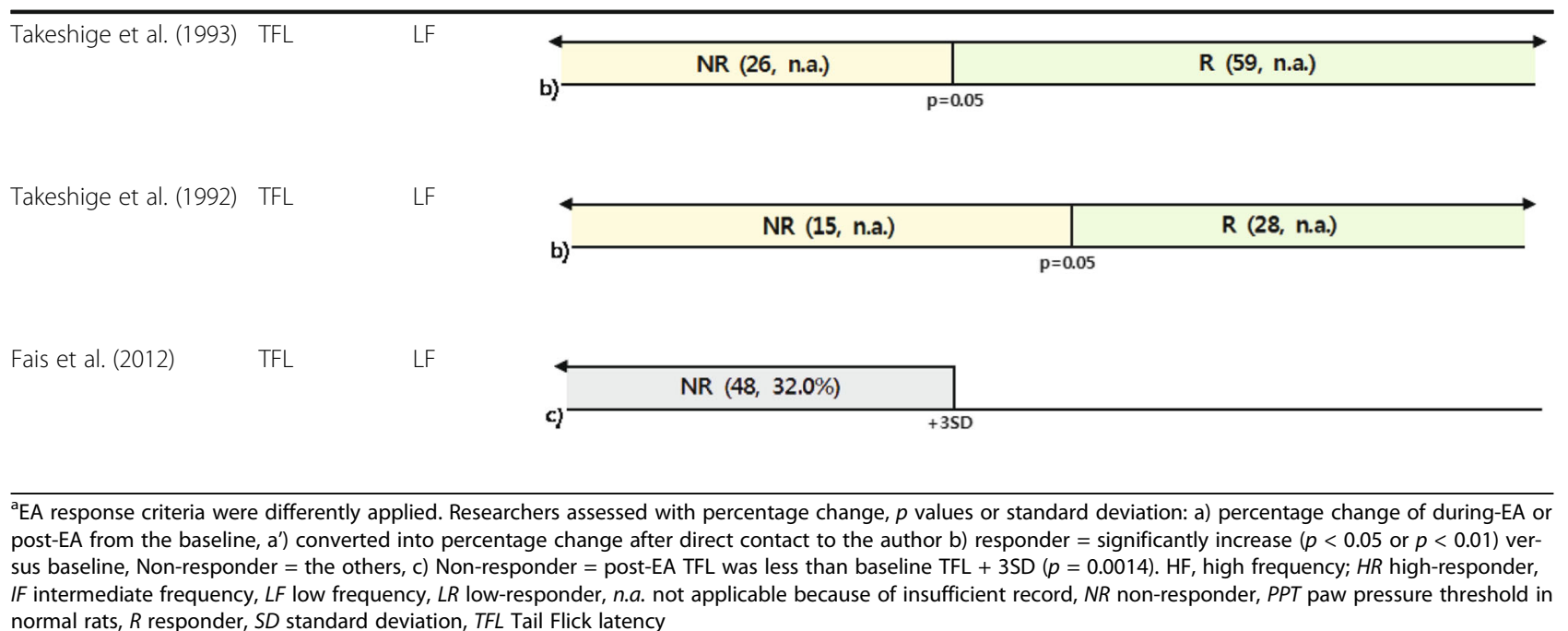

responders [26]. Lee et al. found that CCK A receptorencoding mRNA was highly expressed in the hypothalamus of non-responders and suggested that not only the CCK A receptor; CCK B receptor level per se but also that of the CCK receptor played important roles in the insensitivity to pain modulation afforded by EA [17]. Further, Ko et al. showed that the mRNAs encoding both the CCK A and CCK $\mathrm{B}$ receptor mRNAs were more highly expressed; these results were in contrast to those reported by Lee et al.. The use of different EA frequencies may explain these discrepancies, as Lee et al. delivered EA at $2 \mathrm{~Hz}$, and the EA of Ko et al. was delivered at $100 \mathrm{~Hz}$. Citing the data of Lee et al., Kim et al. found that CCK A receptor-knockout rats enjoyed significantly higher levels of anti-nociceptive effects after $2 \mathrm{~Hz}$ EA. Interestingly, the CCK receptor was more highly expressed in the hypothalamus of the nonresponder group $[17,18,29]$. However, Wang et al. found no difference in the level of CCK receptor expression in the spinal dorsal horns of responders and nonresponders [32] (Tables 4 and 5).

\section{Intrinsic factors affording a good response to acupuncture}

Several intrinsic factors were significant in EA responders. Takeshige et al. showed that the evoked potential in the posterior arcuate nucleus of hypothalamus and the dorsal periaqueductal gray matter of responders differed from that of non-responders [20, 21]. They also reported that administration of morphine (i.p. or microinjection into posterior arcuate nucleus of hypothalamus) increased the anti-nociceptive effects of EA to a level similar to high responders [20], whereas acupuncture-induced anti-nociception in responders remained unchanged. Both modulation of met-enkephalin [21] and $\beta$-endorphin $[20,21]$ could also convert the response of EA.

Hypophysectomy abolished the EA induced antinociception and evoked potentials of the posterior hypothalamic arcuate nucleus in responders (Tables 4 and 5) $[20,21]$. Sekido et al. confirmed that an opioid receptor antagonist, naloxone (i.p.), attenuated EA analgesia in responders (Table 4) [25]. Fais et al. showed that EA combined with inhibition of norepinephrine and serotonin uptake (i.p. and i.t.) converted non-responders into responders (Table 4) [24]. Kim et al. found that hypothalamic 5'-AMP-activated protein kinase (AMPK) gene expression was upregulated after low-frequency EA in responder rats, and hypothalamic microinjection of a dominant-negative AMPK adenovirus, which inhibits AMPK activity, reduced EA analgesia; the wild-type control virus did not do so (Tables 4 and 5) [31].

Additionally, Sekido et al. demonstrated that peripheral inflammation potentiated the EA-induced sensitivity to analgesia; EA responders were about $50 \%$ as sensitive to EA analgesia in normal state, but all rats suffering from inflammation were more sensitive to EA. Naloxone, an opioid receptor antagonist, inhibited EA antinociception in normal rats but decreased EA analgesia in rats with inflamed paws in this study (Table 5) [25].

\section{Intrinsic factors influencing both high- and low-level responsiveness to acupuncture}

Orphanin FQ (OFQ) influenced the response levels depending on the sites of action; intrathecal injection of anti-OFQ antibody converted responders into non-responders, and intracerebral injection of the antibody converted non-responders into responders (Table 5) [27]. 
Table 4 Differentially expressed or changed factors between responder and non-responder

\begin{tabular}{|c|c|c|c|}
\hline Author (Year) & Target regions & Responder > Non-responder & Non-responder > Responder \\
\hline \multicolumn{4}{|l|}{ Low Frequency EA } \\
\hline Kim (2014) & Hypothalamus & AMPK mRNA expression & \\
\hline Wang $(2012)^{a}$ & $\begin{array}{l}\text { Spinal dorsal horn } \\
(\mathrm{L} 5-\mathrm{L} 6)\end{array}$ & $\begin{array}{l}\text { - Neurotransmitter receptors-related genes: } \\
\text { Aplnr, Gabrg2 (at } 24 \mathrm{~h} \text { ) } \\
\text { - Regulation of proinflammatory } \\
\text { cytokines-related genes: Fcgr2b and Gsk3b }\end{array}$ & $\begin{array}{l}\text { - Neurotransmitter receptors-related genes: } \\
\text { Gabrg2 (at } 1 \text { h), and Htr1f } \\
\text { - Proinflammatory cytokines-related gene } \\
\text { expression: C5ar1, IL-6 and TNFa } \\
\text { - Neurotransmitter receptors-related } \\
\text { genes: Gabra2 }\end{array}$ \\
\hline Gao $(2007)^{b}$ & Hypothalamus & $\begin{array}{l}\text { Voltage-gated } \mathrm{K}+\text { channels, solute carrier family } 8 \text {, } \\
\text { Synaptic vesicle glycoprotein } 2 \mathrm{~b} \text {, glutamatergic } \\
\text { A receptor, ghrelin precursor, melanocortin } \\
4 \text { receptor and neuroligin } 1\end{array}$ & \\
\hline Lee (2002) & Hypothalamus & & CCK-AR mRNA expression, but not CCK-BR \\
\hline Takeshige (1993) & Dorsal PAG & Neural activity evoked by EA & \\
\hline Takeshige (1992) & $\begin{array}{l}\text { Medial arcuate nucleus } \\
\text { of hypothalamus }\end{array}$ & Neural activity evoked by EA & \\
\hline \multicolumn{4}{|l|}{ High Frequency EA } \\
\hline Wang $(2012)^{a}$ & $\begin{array}{l}\text { Spinal dorsal horn } \\
\text { (L5-L6) }\end{array}$ & Aplnr, Gabrg2 (at 24 h) & None \\
\hline Ko (2006) & Hypothalamus & & Both CCK-AR and CCK-BR mRNA expression \\
\hline Liu (1999) & Spinal perfusate & & CCK-8-ir \\
\hline
\end{tabular}

a Through CDNA microarray, Wang (2012) compared the responder group, the non-responder group and the restraint group (not applied EA) at $1 \mathrm{~h}$ and $24 \mathrm{~h}$ time point after EA stimulation. We selectively reported genes which were significantly different between the responder group and the non-responder group and a more different group from the restraint group was described as the subject. Almost all genes, cited in this table, showed statistical difference at $1 \mathrm{~h}$ time point and only Gabrg2 was statistically different between the responder and the non-responder group both at $1 \mathrm{~h}$ and at $24 \mathrm{~h}$ time point.

${ }^{b}$ We selectively reported statistically different genes through both microarray and RT-PCR. Gao (2007) conducted dissecting hypothalamus immediately after EA stimulation. AMPK, 5'-AMP-activated protein kinase (regulation of energy homeostasis); CCK-8-ir, cholecystokinin octapeptide like immunoreactivity; CCK-AR, cholecystokinin A receptor; CCK-BR, cholecystokinin B receptor; PAG, periaqueductal central gray

\section{Candidate genes affecting EA response from transcriptomic analyses}

Transcriptomic analyses revealed that gene expression levels in both the dorsal spinal cord and the hypothalamus varied among animals [28, 32]. Wang et al. profiled gene expression in the spinal dorsal horn after application of 2- and $100-\mathrm{Hz}$ EA and found that the levels of mRNAs encoding neurotransmitter receptors (including the Aplnr, GABAA, glycine, and 5-HT1 receptors) were upregulated in responder (but not non-

Table 5 Biological factors that convert the EA response from responder to non-responder or vice versa

\begin{tabular}{|c|c|c|c|}
\hline \multirow[t]{2}{*}{ Author (Year) } & \multirow[t]{2}{*}{ EA } & \multicolumn{2}{|l|}{ Factors } \\
\hline & & Responder $\rightarrow$ Non-responder & Non-responder $\rightarrow$ Responder \\
\hline Kim (2014) & LF & Inhibiting AMPK activity in the hypothalamus & \\
\hline Fais (2012) & LF & & $\begin{array}{l}\text { Inhibitor of norepinephrine and serotonin uptake at spinal } \\
\text { terminals of descending pain inhibitory pathways (amitriptyline, i.p. or i.t) }\end{array}$ \\
\hline Kim (2007) & LF & & CCK-AR KO \\
\hline Sekido (2003) & LF & Naloxone (opioid receptor antagonist, i.p.) & \\
\hline Takeshige (1993) & LF & $\begin{array}{l}\text {-Hypophysectomy } \\
\text {-Antiserum of } \beta \text {-endorphin (i.c.v.) }\end{array}$ & Inhibitor of the degrading enzymes of met-enkephalin (DPA, i.p.) \\
\hline Takeshige (1992) & LF & Hypophysectomy & $\begin{array}{l}\text { - Morphine (i.p.) } \\
\text { - Morphine (into post. Arcuate nucleus) } \\
\text { - } \beta \text {-endorphin (into post. Arcuate nucleus) }\end{array}$ \\
\hline Han (1985) & IF & & CCK-8 AS (i.c.v. or i.t.) \\
\hline Tian (1998) & $\mathrm{HF}$ & OFQ-Ab (i.t.) & OFQ-Ab (i.c.v.) \\
\hline Tang (1997) & $\mathrm{HF}$ & & Antisense CCK (i.c.v.) \\
\hline
\end{tabular}

AMPK 5'-AMP-activated protein kinase (regulation of energy homeostasis), AS antiserum, CCK cholecystokinin, CCK-8 cholecystokinin octapeptide, CCK-AR cholecystokinin A receptor, DPA D-phenylalanine, i.c.v. intracerebroventricular injection, i.p. intraperitoneal injection, i.t. intrathecal injection, KO knockout, Met-Enk methionine enkephalin, OFQ-Ab orphanin FQ anibody, post. Posterior 
responder) rats [32]. They also showed that genes involved in inflammatory modulation were differentially expressed. In non-responder rats, mRNAs encoding proteins associated with the release of proinflammatory cytokines (e.g., IL-6 and TNF- $\alpha$ ) were more highly expressed in non-responders than in responders after $2-\mathrm{Hz}$ EA. However, genes inhibiting the release of proinflammatory cytokines, including Fcgr2b, GSK3b, and Tsc22d3, were upregulated to a greater extent in responders than in non-responders (Table 4) [32].

Gao et al. observed the changes of hypothalamic gene expressions using microarray analysis. They showed that that several genes including a glutamatergic receptor (Grm6), a precursor of ghrelin (Ghrl), the melanocortin 4 receptor $(\mathrm{Mc} 4 \mathrm{r})$, and neuroligin 1 (Nlgn1) were significantly upregulated in the hypothalamus of responders (Table 4) [28].

\section{Discussion}

In this review, we have shown that the levels of signaling molecules associated with acupuncture analgesia (those of the descending inhibitory system, endogenous opioids, and CCK-8 and receptors thereof) may be differentially expressed in responders and non-responders. Also, modulation of such factors may change the response to EA. Responders and non-responders differ in terms of AMPK expression in the hypothalamus and in the levels of neurotransmitter receptors and pro-inflammatory cytokines genes in the spinal cord $[31,32]$ and such differences may also affect response related to EA (Fig. 2).
Acupuncture analgesia is an integrative process involving both afferent impulses from the acupoints and the painful region [12]. In an early responder study, Takeshige et al. suggested that afferent neural transmission could explain different responses to EA [20, 21]. Upon application of EA, the changes in the neuronal activity in the arcuate nucleus and dorsal PAG differed between responders and non-responders and correlated with the extent of EA-induced analgesia. EA increased neuronal activity in the PAG of responders, irrespective of whether EA was applied ipsilaterally or contralaterally.

As mentioned above, the endogenous descending inhibitory system of the central nervous system (CNS) and various signaling molecules, including opioid peptides, glutamate, and 5-hydroxytryptamine, contribute to acupuncture-induced analgesia [33]. The release of opioid peptides during acupuncture is frequencydependent. Low- and high-frequency EA release enkephalin/ $\beta$-endorphin and dynorphin, respectively, in the CNS [34]. Responder analysis has also shown that differences in the expression levels of these factors contribute to individual differences in sensitivity to EA analgesia. Sekido et al. found that intraperitoneal injection of naloxone converted responders to low-frequency EA into non-responders [25]. Injection of anti- $\beta$-endorphin antibody into the cerebral ventricles abolished the analgesic effects of low-frequency EA in responders (21). $D$-phenylalanine, an inhibitor of enzymes degrading met-enkephalin, morphine, and $\beta$-endorphin changed the neuronal activity induced by low-frequency EA in non-responders to that characteristic of responders $[20$,

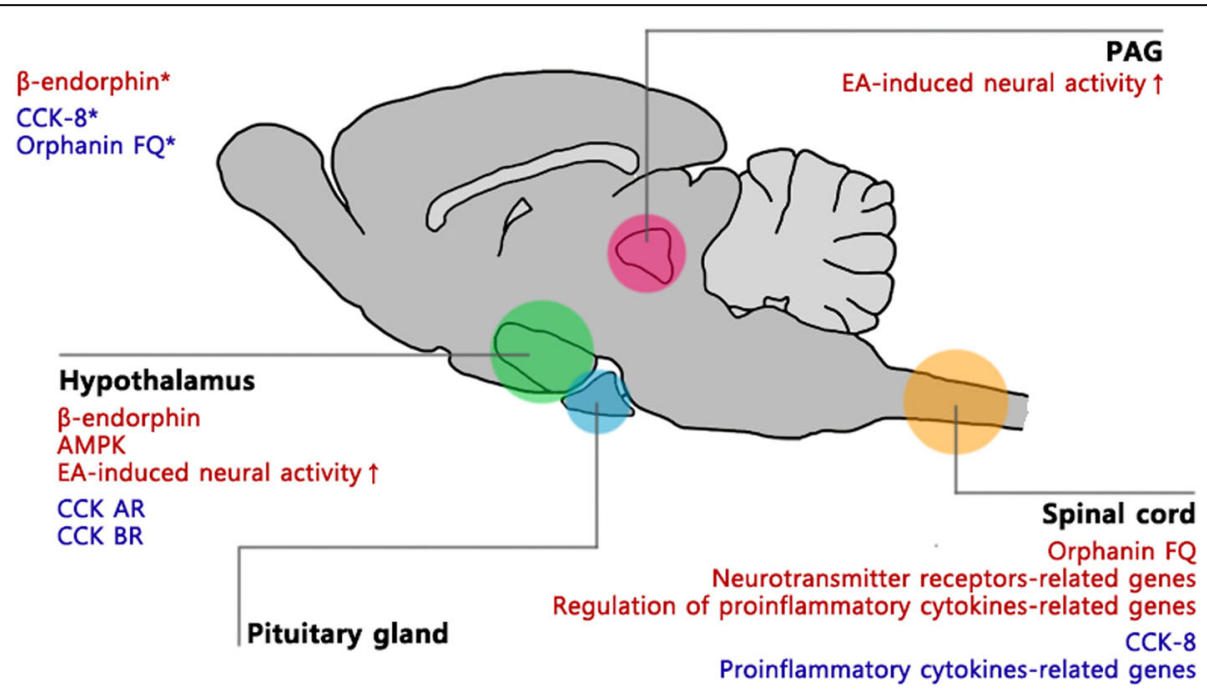

Fig. 2 A scheme of EA responsiveness-related factors and regions. The indicated specific regions, hypothalamus, PAG, pituitary gland and spinal cord, are well-known to be associated with pain regulation, and have been reconfirmed through researches on EA responsiveness. An asterisk implies intracerebroventricular injection. Responder-related factors and non-responder-related factors have been grouped in red and blue letters respectively. CCK AR, cholecystokinin A receptor; CCK BR, cholecystokinin B receptor; CCK-8, cholecystokinin octapeptide; Hypo, hypothalamus; PAG, periaqueductal grey; Pit. g., pituitary gland 
21]. The OFQ peptide is involved in many physiological processes, including pain. The effects of the peptide on the nervous system are complex; it is generally accepted that spinal OFQ is anti-nociceptive, while OFQ exerts an anti-opioid action and causes hyperalgesia when injected supraspinally [35]. Tian et al. explored whether OFQ modulated the responses of EA and found that intrathecal injection of an anti-OFQ antibody converted responders to non-responders, whereas intracerebral injection had the reverse effect [27].

Additionally, the norepinephrine and serotonin systems of the descending inhibitory pathway contribute to individual differences in EA analgesia. Fais et al. found that the poor analgesia of non-responders changed to the level of analgesia enjoyed by responders after administration (i.p. and i.t.) of inhibitors of norepinephrine and serotonin uptake at the spinal terminals of the descending inhibitory pathways [24].

Kim et al. suggested that hypothalamic AMPK might be an intrinsic mediator of the EA response [31]. The AMPK enzyme plays roles in cellular energy homeostasis and metabolic stress and was recently shown to modulate both acute and chronic neuropathic pain [36, 37]. Hypothalamic AMPK gene expression was upregulated after low-frequency EA in responder rats, and hypothalamic inhibition of AMPK activity using dominantnegative AMPK adenovirus reduced EA analgesia.

The best-studied intrinsic factors are CCK- 8 and receptors thereof. CCK is a CNS neurotransmitter playing roles in pain, satiety, feeding, learning, cognition, and emotion. CCK-8, a major form of CCK, is found predominantly in the CNS [38-40], and has been found to play a role in the development of tolerance to EA; CCK8 may counter pain alleviation by exerting an anti-opioid action [41]. Liu et al. showed that the extent of CCK-8 immunoreactivity increased in the spinal perfusate of non-responders after high-frequency EA [26]. Intracerebroventricular or intrathecal injection of anti-CCK-8 antibody or antisense CCK-8 converted non-responders into responders [19]. However, the levels of CCK in the spinal dorsal horn were increased in both responders and non-responders after EA [32]. It has been suggested that the CCK receptor isoforms, CCK AR and BR are differentially affected [29]. However, it remains unclear which receptor may be more important in terms of the response to EA analgesia. Upon application of lowfrequency EA, the level of CCK AR but not BR mRNA increased [17]. CCK AR-knockout rats exhibited an elevated anti-nociceptive response after low-frequency EA [18]. In contrast, the hypothalamic levels of mRNAs encoding both CCK AR and BR were upregulated after high-frequency EA. A CCK-B antagonist potentiated EA anti-nociception after high-frequency EA [42]. However, no significant changes in the levels of CCK AR or BR
mRNAs were evident in the dorsal horn of the spinal cord [32]. Thus, it seems clear that the CCK system reduces the analgesic effects of EA, regardless of the frequency. However, further research is required to determine whether this is the principal cause of EA nonresponsiveness.

Transcriptomic analyses have suggested that the actions of several novel intrinsic factors may explain the different responses to acupuncture. Wang et al. found that the differential regulation of genes encoding neurotransmitters and their receptors indicated that the neurotransmitter system may be more active in responders than in non-responders. Genes involved in the modulation of inflammation were also differentially expressed. In non-responder rats, the levels of mRNAs facilitating the release of proinflammatory cytokines (including IL-6 and TNF- $\alpha$ ) were higher in nonresponders. In responders, the levels of mRNAs inhibiting the release of proinflammatory cytokines (e.g., Fcgr2b, GSK3b, and Tsc22d3) were higher in responders. It is known that proinflammatory cytokines induce the release of various inflammatory materials and play important roles in the nociceptive and analgesic systems of the CNS. These results suggest that the intrinsic response to spinal cord inflammation may partly explain the different responses to EA [32].

There are several issues to discuss for developing the responder analysis more useful. First, the reproducibility of the EA response is an important consideration. If factors influencing this response are intrinsic, the response must be reproducible. Wang et al. repeated TFL tests on the same rats at 2-day intervals and found that the EA response was maintained [32]. However, most studies have not addressed reproducibility. Additionally, more than $70 \%$ of the included studies measured antinociceptive effects in non-diseased normal animals, and it is doubtful that results can be applicable to diseased individuals. Kim et al. explored whether the antinociceptive response to EA in non-diseased normal animals was reproduced after induction of neuropathic pain; the responses of both responders and nonresponders were in fact maintained [18]. However, Sekido et al. reported a different finding: $50 \%$ of nondiseased normal rats were classified as EA responders, but all tested rats, regardless of response status, experienced EA-induced analgesia of carrageenan-induced inflammatory pain [25]. In addition, it should be considered that the ratio of responder and non-responder might be varied across the types of disease. In Li et al.'s study, the number of responder was twice as many as that of non-responder for the modulatory effects of EA (acupoint PC5-PC6) on cardiovascular reflex response [43], while there are not enough data for the responder ratio in other physiological or pathological condition. 
Further studies with different pathological models should address this question.

Second, the classification of responders or nonresponders needs to set a good validation. The cut-off criteria for responders were extremely variable among the 14 relevant studies, including a $10-150 \%$ increase in the TFL or the pressure pain threshold from baseline. The notifying accurate description of classifying responder or non-responder and the use of validated cut-offs is essential in future work. Next, the acupuncture conditions reviewed are very limited; all studies employed very common methods such as EA of acupoint ST36 (100\% of studies). There are more than 360 acupoints with various stimulation methods [44]. Thus, caution is required when generalizing the results.

Further, the responder studies in clinical setting are limited. Only a few studies have sought to identify human responders. Chae et al. used a microarray to identify genes differing in expression level between high- and low-responders in 15 healthy volunteers. Genes related with signaling, stress response, transcriptional regulators, and/or regulators of immune function may be relevant, although the "responder" mechanism needs to be clarified further [45]. Genetic factors have been suggested to play roles in individual sensitivity to acupuncture $[5,13$, 45]. However, the gap between pre-clinical and clinical researches remains very wide, and further translational clinical studies are essential.

Next, various parameters beyond individual variance can affect the responsiveness of acupuncture. For instance, the selection of acupoints, the number of acupuncture treatments, stimulation modality (manual, EA or others) and intensity, learning from pre-exposed cues, and the condition of the patients can influence the acupuncture effects [34, 46-50]. EA tolerance, which means a gradual decline of EA effects due to the repeated use, is also important to explain the lowresponsiveness of EA [51]. It might be interesting how these various factors synergistically contribute to acupuncture effects.

Finally, it is also important to consider how the various intrinsic factors identified can contribute to the elucidating the acupuncture mechanism. Though there are still lots of limitation, the existed evidences suggest this possibility: the factors identified in the low responder (i.e. CCK-8 and spinal OFQ) have shown to be involved in the attenuation of EA analgesia and the induction of EA tolerance [19, 26, 27]; the injection of morphine and $\beta$-endorphin could convert nonresponder into high-responder, and these results are helpful to understand the involvement of endogenous opioid system in the acupuncture analgesia [20, 21]. Further well designed responder analysis is needed to aid the acupuncture mechanism.
In spite of the several limitations mentioned above, our review of pre-clinical studies is meaningful to show the benefit of responder and non-responder analysis for elucidating the mechanism of acupuncture in pain. Further responder analysis considering the validated cut-off criteria, animal models depicting human disease, and real-world acupuncture methods is recommendable. This kind of approach might help to develop the strategy to enhance the acupuncture effects in pain medicine.

\section{Conclusion}

In conclusion, we confirmed that dividing animals into responders and non-responders identified novel candidate mediators of the effects of acupuncture. A number of studies have found that the EA responsiveness could be modifiable by modulating these mediators. Although most included studies regarding EA responsiveness was investigated in the physiological state, and there are several methodological issues to be improved, this study may allow us the development of new strategies potentiating the therapeutic effects of acupuncture.

\section{Additional file}

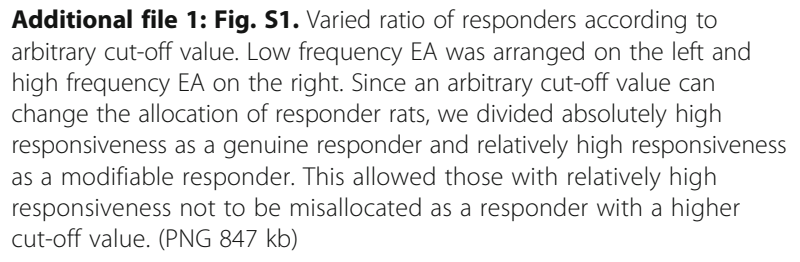

\section{Abbreviations}

AMPK: 5'-AMP-activated protein kinase; CCK AR: cholecystokinin A receptor: CCK BR: cholecystokinin B receptor; CCK: cholecyctokinin; CCK-8: cholecystokinin octapeptide; CNS: central nervous system; EA: electroacupuncture;

OFQ: Orphanin FQ; PAG: periaqueductal grey; TFL: tail flick latency

\section{Acknowledgments}

The English in this review has been checked by at least two professional editors of Textcheck, both native speakers of English (http://www.textcheck.com/ certificate/hsqock). This research was supported by the National Research Foundation of Korea funded by the Korean Ministry of Science

(NRF-2015M3A9E052338 and 2017R1A2B4009963).

\section{Funding}

This research was supported by the National Research Foundation of Korea funded by the Korean Ministry of Science (NRF-2015M3A9E052338 and 2017R1A2B4009963).

\section{Availability of data and materials}

The data sets supporting the conclusions of this article are included within the article.

\section{Authors' contributions}

Conceived and designed this review: HJP MY DHH YC HL. Extracted the data: YKK JYP SNK. Analyzed the data: YKK JYP SL JYO. Wrote the paper: YKK HJP. All authors read and approved the final manuscript.

Competing interests

The authors declare that they have no competing interests. 


\section{Consent for publication}

This information is not relevant.

\section{Ethics approval and consent to participate}

Not applicable.

\section{Publisher's Note}

Springer Nature remains neutral with regard to jurisdictional claims in published maps and institutional affiliations.

\section{Author details}

'Department of Science in Korean Medicine, Graduate School, Kyung Hee University, 26 Kyungheedae-ro, Dongdaemoon-gu, Seoul 02447, Republic of Korea. ${ }^{2}$ College of Korean Medicine, Daejeon University, 62 Daehak-ro, Dong-gu, Daejeon 34520, Republic of Korea. ${ }^{3}$ College of Korean Medicine, Dongguk University. 32, Dongguk-ro, Ilsandong-gu, Goyang-si, Gyeonggi-do 10326, Republic of Korea. ${ }^{4}$ Acupuncture \& Meridian Science Research Center, Kyung Hee University, 26 Kyungheedae-ro, Dongdaemoon-gu, Seoul 02447, Republic of Korea. ${ }^{5}$ Department of Acupuncture and Moxibustion, College of Korean Medicine, Kyung Hee University, Seoul 130-701, Republic of Korea.

Received: 6 December 2016 Accepted: 15 May 2017

\section{Published online: 25 May 2017}

\section{References}

1. Gereau RW, Sluka KA, Maixner W, Savage SR, Price TJ, Murinson BB, et al. A pain research agenda for the 21st century. J Pain. 2014;15(12):1203-14

2. Melnikova I. Pain market. Nat Rev Drug Discov. 2010;9(8):589-90.

3. Mills S, Torrance N, Smith BH. Identification and Management of Chronic Pain in primary care: a review. Curr Psychiatry Rep. 2016;18(2):22.

4. Tsang A, Von Korff M, Lee S, Alonso J, Karam E, Angermeyer MC, et al. Common chronic pain conditions in developed and developing countries: gender and age differences and comorbidity with depression-anxiety disorders. J Pain. 2008:9(10):883-91.

5. Ren $Z Y, X u X Q, B a o Y P, H e J$, Shi L, Deng JH, et al. The impact of genetic variation on sensitivity to opioid analgesics in patients with postoperative pain: a systematic review and meta-analysis. Pain Physician. 2015;18(2):131-52.

6. Scholz J, Woolf CJ. Can we conquer pain? Nat Neurosci. 2002;5(Suppl):1062-7.

7. Schork NJ. Time for one-person trials. Nature. 2015;520(7549):609-11.

8. Woodcock J, Witter J, Dionne RA. Stimulating the development of mechanism-based, individualized pain therapies. Nat Rev Drug Discov. 2007; 6(9):703-10

9. Kim TH, Kang JW, Kim KH, Kang KW, Shin MS, Jung SY, et al. Acupuncture for the treatment of dry eye: a multicenter randomised controlled trial with active comparison intervention (artificial teardrops). PLoS One. 2012;7(5): e36638.

10. Lau CH, Wu X, Chung VC, Liu X, Hui EP, Cramer H, et al. Acupuncture and related therapies for symptom Management in Palliative Cancer Care: systematic review and meta-analysis. Medicine (Baltimore). 2016;95(9):e2901.

11. Qin Z, Wu J, Zhou J, Liu Z. Systematic review of acupuncture for chronic prostatitis/chronic pelvic pain syndrome. Medicine (Baltimore). 2016;95(11):e3095.

12. Zhang R, Lao L, Ren K, Berman BM. Mechanisms of acupunctureelectroacupuncture on persistent pain. Anesthesiology. 2014;120(2):482-503.

13. Park HJ, Kim ST, Yoon DH, Jin SH, Lee SJ, Lee HJ, et al. The association between the DRD2 Taql a polymorphism and smoking cessation in response to acupuncture in Koreans. J Altern Complement Med. 2005;11(3):401-5.

14. Li K, Zhang Y, Ning Y, Zhang H, Liu H, Fu C, et al. The effects of acupuncture treatment on the right frontoparietal network in migraine without aura patients. J Headache Pain. 2015;16:518.

15. Salehi A, Marzban M, Imanieh MH. The evaluation of curative effect of acupuncture: a review of systematic and meta-analysis studies. J Evid Based Complementary Altern Med. 2015;

16. Li P, Ayannusi O, Reid C, Longhurst JC. Inhibitory effect of electroacupuncture (EA) on the pressor response induced by exercise stress. Clin Auton Res. 2004;14(3):182-8.

17. Lee $G$, Rho S, Shin M, Hong M, Min B, Bae H. The association of cholecystokinin-a receptor expression with the responsiveness of electroacupuncture analgesic effects in rat. Neurosci Lett. 2002;325(1):17-20.

18. Kim SK, Moon HJ, Park JH, Lee G, Shin MK, Hong MC, et al. The maintenance of individual differences in the sensitivity of acute and neuropathic pain behaviors to electroacupuncture in rats. Brain Res Bull. 2007:74(5):357-60.

19. Han JS, Ding XZ, Fan SG. Is cholecystokinin octapeptide (CCK-8) a candidate for endogenous anti-opioid substrates? Neuropeptides. 1985;5(4-6):399-402.

20. Takeshige C, Nakamura A, Asamoto S, Arai T. Positive feedback action of pituitary beta-endorphin on acupuncture analgesia afferent pathway. Brain Res Bull. 1992;29(1):37-44.

21. Takeshige C, Oka K, Mizuno T, Hisamitsu T, Luo CP, Kobori M, et al. The acupuncture point and its connecting central pathway for producing acupuncture analgesia. Brain Res Bull. 1993;30(1-2):53-67.

22. Lu L, Zhang XG, Zhong LL, Chen ZX, Li Y, Zheng GQ, et al. Acupuncture for neurogenesis in experimental ischemic stroke: a systematic review and meta-analysis. Sci Rep. 2016;6:19521.

23. Macleod MR, O'Collins T, Howells DW, Donnan GA. Pooling of animal experimental data reveals influence of study design and publication bias. Stroke. 2004;35(5):1203-8.

24. Fais RS, Reis GM, Rossaneis AC, Silveira JW, Dias OM, Prado WA Amitriptyline converts non-responders into responders to low-frequency electroacupuncture-induced analgesia in rats. Life Sci. 2012;91(1-2):14-9.

25. Sekido R, Ishimaru K, Sakita M. Differences of electroacupuncture-induced analgesic effect in normal and inflammatory conditions in rats. Am J Chin Med. 2003;31(6):955-65.

26. Liu SX, Luo F, Shen S, Yu YX, Han JS. Relationship between the analgesic effect of electroacupuncture and CCK-8 content in spinal perfusate in rats. Chin Sci Bull. 1999;44(3):240-3.

27. Tian JH, Zhang W, Fang Y, Xu W, Grandy DK, Han JS. Endogenous orphanin FQ: evidence for a role in the modulation of electroacupuncture analgesia and the development of tolerance to analgesia produced by morphine and electroacupuncture. Br J Pharmacol. 1998;124(1):21-6.

28. Gao YZ, Guo SY, Yin QZ, Hisamitsu T, Jiang XH. An individual variation study of electroacupuncture analgesia in rats using microarray. Am J Chin Med. 2007:35(5):767-78

29. Ko ES, Kim SK, Kim JT, Lee G, Han JB, Rho SW, et al. The difference in mRNA expressions of hypothalamic CCK and CCK-A and -B receptors between responder and non-responder rats to high frequency electroacupuncture analgesia. Peptides. 2006;27(7):1841-5.

30. Tang NM, Dong HW, Wang XM, Tsui ZC, Han JS. Cholecystokinin antisense RNA increases the analgesic effect induced by electroacupuncture or low dose morphine: conversion of low responder rats into high responders. Pain. 1997;71(1):71-80

31. Kim SK, Sun B, Yoon H, Lee JH, Lee G, Sohn SH, et al. Expression levels of the hypothalamic AMPK gene determines the responsiveness of the rats to electroacupuncture-induced analgesia. BMC Complement Altern Med. 2014;14:211.

32. Wang $K$, Zhang $R$, Xiang $X$, He F, Lin L, Ping $X$, et al. Differences in neuralimmune gene expression response in rat spinal dorsal horn correlates with variations in electroacupuncture analgesia. PLoS One. 2012;7(8):e42331.

33. Zhao ZQ. Neural mechanism underlying acupuncture analgesia. Prog Neurobiol. 2008;85(4):355-75

34. Han JS. Acupuncture: neuropeptide release produced by electrical stimulation of different frequencies. Trends Neurosci. 2003;26(1):17-22.

35. Di Cesare ML, Micheli L, Ghelardini C. Nociceptin/orphanin FQ receptor and pain: feasibility of the fourth opioid family member. Eur J Pharmacol. 2015; 766:151-4.

36. Melemedjian OK, Asiedu MN, Tillu DV, Sanoja R, Yan J, Lark A, et al. Targeting adenosine monophosphate-activated protein kinase (AMPK) in preclinical models reveals a potential mechanism for the treatment of neuropathic pain. Mol Pain. 2011;7:70.

37. Tillu DV, Melemedjian OK, Asiedu MN, Qu N, De Felice M, Dussor G, et al. Resveratrol engages AMPK to attenuate ERK and mTOR signaling in sensory neurons and inhibits incision-induced acute and chronic pain. Mol Pain. 2012;8:5.

38. Crawley JN, Corwin RL. Biological actions of cholecystokinin. Peptides. 1994; 15(4):731-55.

39. Dockray GJ. Cholecystokinin and gut-brain signalling. Regul Pept. 2009; 155(1-3):6-10

40. Zhang JG, Liu JX, Jia XX, Geng J, Yu F, Cong B. Cholecystokinin octapeptide regulates the differentiation and effector cytokine production of CD4(+) $T$ cells in vitro. Int Immunopharmacol. 2014;20(2):307-15.

41. Carlino E, Benedetti F. Different contexts, different pains, different experiences. Neuroscience. 2016;

42. Zhou Y Sun YH, Shen JM, Han JS. Increased release of immunoreactive CCK-8 by electroacupuncture and enhancement of electroacupuncture 
analgesia by CCK-B antagonist in rat spinal cord. Neuropeptides. 1993; 24(3):139-44

43. Li M, Tjen ALSC, Guo ZL, Longhurst JC. Electroacupuncture modulation of reflex hypertension in rats: role of cholecystokinin octapeptide. Am J Physiol Regul Integr Comp Physiol. 2013;305(4):R404-13.

44. WHO Western Pacific Region. WHO standard acupuncture point locations in the western Pacific region. In: WHO standard acupuncture point locations in the Western Pacific region; 2008.

45. Chae Y, Park HJ, Hahm DH, Yi SH, Lee H. Individual differences of acupuncture analgesia in humans using CDNA microarray. J Physiol Sci. 2006;56(6):425-31.

46. Lee J, Napadow V, Park K. Pain and sensory detection threshold response to acupuncture is modulated by coping strategy and acupuncture sensation. BMC Complement Altern Med. 2014;14:324.

47. Liu H, Xu JY, Li L, Shan BC, Nie BB, Xue JQ. FMRI evidence of acupoints specificity in two adjacent acupoints. Evid Based Complement Alternat Med. 2013;2013:932581.

48. MacPherson H, Maschino AC, Lewith G, Foster NE, Witt CM, Vickers AJ. Characteristics of acupuncture treatment associated with outcome: an individual patient meta-analysis of 17,922 patients with chronic pain in randomised controlled trials. PLoS One. 2013;8(10):e77438.

49. Prady SL, Burch J, Vanderbloemen L, Crouch S, MacPherson H. Measuring expectations of benefit from treatment in acupuncture trials: a systematic review. Complement Ther Med. 2015;23(2):185-99.

50. Bossut DF, Mayer DJ. Electroacupuncture analgesia in rats: naltrexone antagonism is dependent on previous exposure. Brain Res. 1991;549(1):47-51.

51. Cui L, Ding Y, Feng Y, Chen $S, X u Y$, Li M, et al. MiRNAs are involved in chronic electroacupuncture tolerance in the rat hypothalamus. Mol Neurobiol. 2017;54(2):1429-39.

\section{Submit your next manuscript to BioMed Central and we will help you at every step:}

- We accept pre-submission inquiries

- Our selector tool helps you to find the most relevant journal

- We provide round the clock customer support

- Convenient online submission

- Thorough peer review

- Inclusion in PubMed and all major indexing services

- Maximum visibility for your research

Submit your manuscript at www.biomedcentral.com/submit
C Biomed Central 